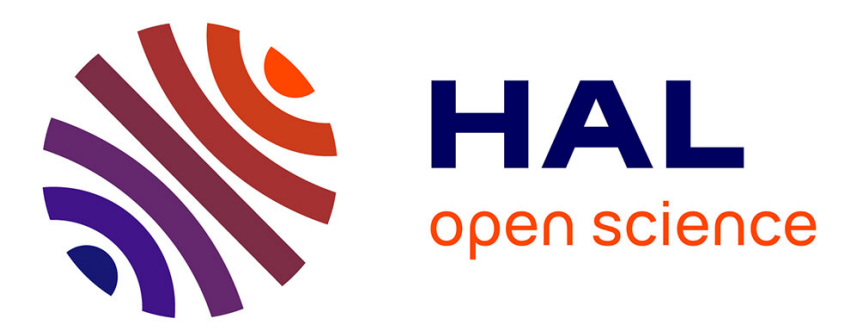

\title{
L'élevage ovin montmorillonnais partagé entre l'intensification et une voie plus extensive. Observations pour un débat $(1)$
}

M. Benoit, G. Laignel, G. Liénard

\section{- To cite this version:}

M. Benoit, G. Laignel, G. Liénard. L'élevage ovin montmorillonnais partagé entre l'intensification et une voie plus extensive. Observations pour un débat(1). Productions Animales, 1991, 4 (5), pp.343359. hal-00895953

\section{HAL Id: hal-00895953 https://hal.science/hal-00895953}

Submitted on 1 Jan 1991

HAL is a multi-disciplinary open access archive for the deposit and dissemination of scientific research documents, whether they are published or not. The documents may come from teaching and research institutions in France or abroad, or from public or private research centers.
L'archive ouverte pluridisciplinaire HAL, est destinée au dépôt et à la diffusion de documents scientifiques de niveau recherche, publiés ou non, émanant des établissements d'enseignement et de recherche français ou étrangers, des laboratoires publics ou privés. 
INRA Prod. Anim., 1991, 4 (5), 343 - 359
M. BENOIT, G. LAIGNEL et G. LIÉNARD

INRA

Laboratoire d'Economie de l'Elevage

Theix - 63122 Saint-Genès-Champanelle
L'élevage ovin montmorillonnais partagé entre l'intensification et une voie plus extensive. Observations pour un débat ${ }^{(1)}$

\begin{abstract}
Dans les années soixante-dix, l'élevage ovin français a bénéficié de rapports de prix favorables entre ses ventes et ses achats de facteurs de production. Mais depuis les années quatre-vingts, la libération des échanges dans le cadre de la P.A.C., a fait passer l'offre de viande ovine française d'un léger déficit plutôt favorable aux producteurs

à une situation où les importations sont en passe de devenir prépondérantes : celles-ci ont atteint $46 \%$ de la consommation en 1990, contre $20 \%$ en 1980 - 1983. Le marché devient directement dépendant des prix des exportateurs anglais et irlandais. Le prix moyen à la production a ainsi diminué de $40 \%$ en francs constants entre 1980 et 1990. Dans un tel contexte, les systèmes ovins les plus intensifs, très consommateurs d'intrants, qui avaient été mis au point lorsque la conjoncture était favorable, peuvent être remis en cause. Mais des systèmes moins intensifs peuvent-ils être adaptés à la nouvelle situation, et dans quelles conditions? La comparaison des différents systèmes ovins pratiqués en Montmorillonnais, dans des élevages ayant des effectifs importants ( 300 brebis et 430 agneaux par travailleur en moyenne), nettement supérieurs à la moyenne française actuelle, peut contribuer aux débats qui s'ouvrent sur ces questions.
\end{abstract}

\title{
Résumé
}

\begin{abstract}
Soumis à une forte concurrence de la part de la Grande-Brelagne et de l'Irlande, l'élevage ovin français connaît des conditions difficiles, marquées par une hausse continue des importations et une baisse des prix payés aux éleveurs (- $40 \%$ entre 1980 et 1990$)$. Les systèmes ovins les plus intensifs, très consommateurs d'intrants, peuvent être remis en cause, mais peuvent-ils être remplacés, et dans quelles conditions, par des systèmes moins intensifs?

L'étude concerne des exploitations ovines du Montmorillonnais (Vienne, France) ayant des effectifs relativement importants dans les conditions françaises (300 brebis et $\mathbf{4 3 0}$ agneaux par travailleur). Sept systèmes de production différents ont pu être identifiés. Deux situations stabilisées correspondant à des options d'intensification nettement différentes sont comparées. Les "Intensifs Réussis " pratiquent un système permettant de produire à la fois des cultures de vente et des ovins, grâce à un chargement des surfaces fourragères élevé (1,5 UGB/ha) et l'engraissement des $2 / 3$ des agneaux en bergerie, l'ensemble entraînant une forte consommation d'engrais (110 unités de $\mathrm{N}+\mathrm{P}+\mathrm{K} / \mathrm{ha}$ ) et de concentrés $(190 \mathrm{~kg} / \mathrm{brebis})$. Les « Herbagers Autonomes " ont opté pour un système davantage basé sur l'herbe (les $3 / 4$ des agneaux sont mis à l'herbe) avec un chargement plus faible (1 UGB/ha) et une plus faible consommation d'intrants (58 NPK/ha et $107 \mathrm{~kg}$ de concentré/brebis) ; mais presque toule la surface est consacrée aux ovins et la production d'agneaux est légèrement plus importante (430 contre 410 par travailleur), avec moins de charges et moins de capital. Les résultats économiques obtenus sont équivalents en 1989 - 1990. Mais les élevages spécialisés moins intensifs, à condition d'être aussi bien gérés que les exploitations intensives, semblent mieux placés face aux aléas. Notamment le nombre d'agneaux produits par brebis doit être maintenu pour permettre l'obtention d'une marge supérieure grâce à la réduction des charges.
\end{abstract}


Le Montmorillonnais, au Sud de la Vienne, est une région peu peuplée où l'élevage ovin est actuellement prépondérant, malgré les difficultés qu'il y rencontre du fait de l'irrégularité des pluies et surtout de la sensibilité de la plupart des sols à la sécheresse et à l'excès d'eau.

Les grandes exploitations ayant peu de main d'œuvre ont introduit, dans les années trente, un système ovin très extensif (de l'ordre de 3 brebis par ha SFP), peu exigeant en capital, basé sur l'herbe, la race Charmoise et le plein air hivernal. La spécialisation ovine a gagné peu à peu l'ensemble des exploitations, mais la conduite très extensive s'est révélée inadaptée aux exploitations moyennes et petites qui ont dû adopter un système moins extrême avec bergerie, des chargements un peu plus élevés ( 4 à 6 brebis/ha) et une plus grande productivité des brebis $(1,2-1,3$ agneaux par brebis et par an contre 0,8 - 0,9). Progressivement, la hausse des charges foncières et plus généralement des charges de structure a amené, dans les années soixante-dix, beaucoup d'éleveurs, notamment des migrants, à intensifier davantage leur production ovine. A partir des années quatrevingts, la diminution progressive des prix des ovins, en francs constants, a mis en difficulté les exploitations les plus endettées ; on commence à diversifier la production avec des bovins ou plus souvent avec des cultures de vente, en particulier après drainage. En 1985, Y. Jean faisait déjà état d'un nouveau débat entre intensification et extensification, spécialisation et diversité (Jean 1985).

Depuis, la conjoncture ovine s'est encore dégradée. En 1990, le prix moyen annuel des ovins à la production (IPPAP) est revenu à l'indice 112 par rapport à 1980, ce qui représente une baisse de $40 \%$ en francs constants. Aucune production, pas même les céréales et les poulets n'en sont à ce niveau. Et aujour-

Figure 1. Situation géographique de l'observatoire ovin du Montmorillonnais. d'hui, les éleveurs et leurs techniciens se demandent quelles orientations prendre. Cette question est générale et se pose dans beaucoup de régions d'élevage ovin (Blanchemain 1988; Boutonnet et Tchamitchian 1990; Durazo et Lossouarn 1990 ; Hubert 1990).

Dans le cadre d'une AIP INRA, réunissant agronomes, zootechniciens et économistes (laboratoires ESR de Grignon et de Theix), un observatoire économique d'exploitations ovines a été mis en place en 1987, en collaboration avec les associations professionnelles locales (GEHP, CGER, Chambre d'Agriculture, Alliance Pastorale, RNED régional ovin).

L'objectif initial est un suivi technique et économique sur plusieurs années d'exploitations ovines $(n=27)$ disposant de structures et d'effectifs de troupeaux supérieurs à la moyenne (de 350 à 1100 brebis), ayant acquis un bon niveau de technicité et pouvant représenter ce vers quoi sont susceptibles d'évoluer les exploitations pérennes. Beaucoup font partie des structures de développement techniques et économiques, mais ce n'est pas le cas de toutes, comme l'ont souhaité les membres du comité de pilotage initial. L'ensemble recouvre une certaine diversité, dont l'analyse peut être utile lorsqu'il s'agit de redéfinir des oriemations adaptatives ou nouvelles. Les résultats économiques présentés dans cette étude ne sont évidemment pas statistiquement représentatifs de ceux des éleveurs ovins de cette région et ni de la moyenne française. Tel n'est pas l'objectif, pour lequel d'autres observations appropriées existent, (comptes de l'agriculture ou RICA (Chaalali 1990)). Les éleveurs de l'échantillon ont, pour la plupart, des résultats supérieurs à l'ensemble. Cependant les difficultés économiques que ces éleveurs rencontrent sont significatives de celles de l'ensemble du secteur.

Après une présentation de l'échantillon dans son ensemble, nous proposons une analyse des logiques de fonctionnement des exploitations qui peuvent être distinguées, en tenant compte

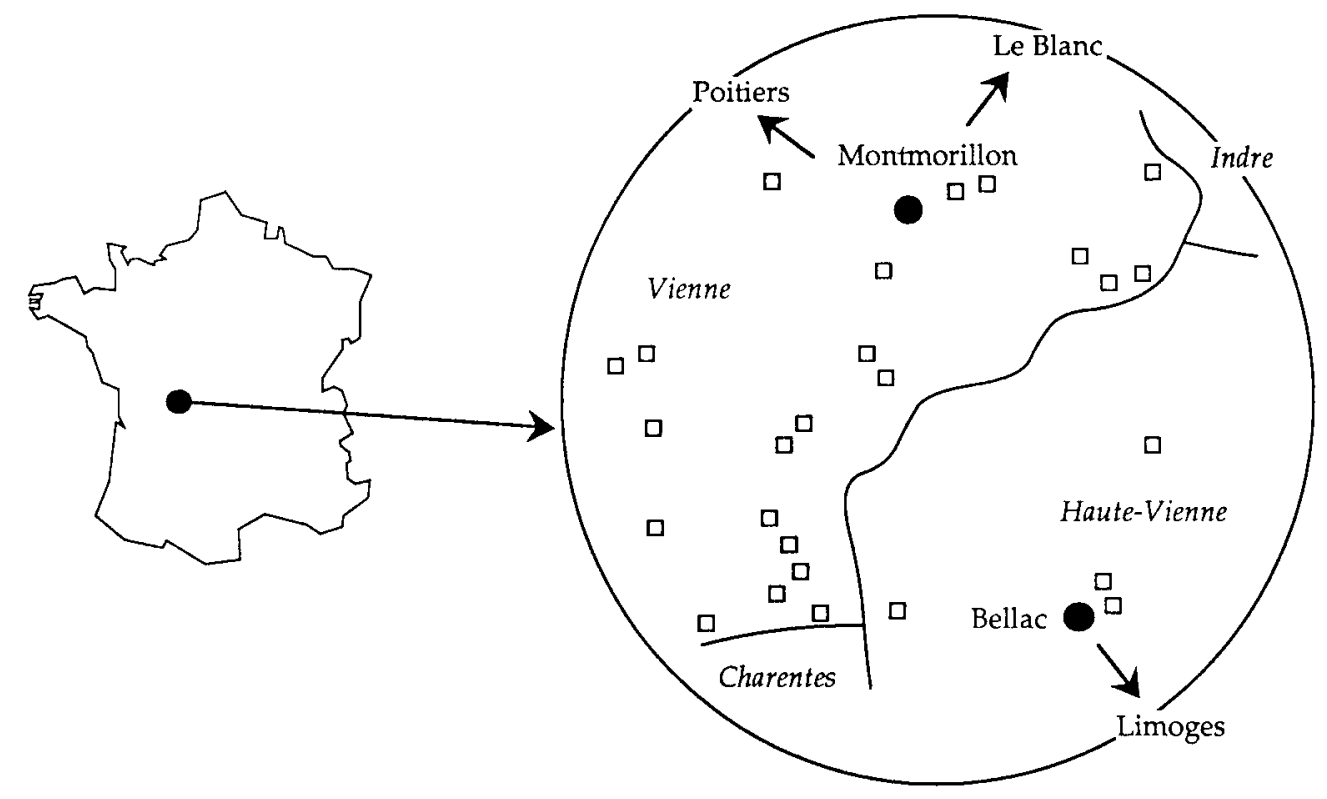


simultanément des structures, des grandes orientations productives, du mode de conduite du troupeau ovin et du niveau d'intensification des surfaces fourragères avec la possibilité de préciser les résultats techniques et économiques obtenus. Les résultats présentés concernent la campagne 1989, mais en tenant compte de ceux de la campagne précédente et de la campagne suivante.

\section{1 / Présentation de l'échantillon}

\section{1 / Localisation}

Les 27 exploitations sont localisées dans le Sud de la Vienne, entre Montmorillon au Nord, Bellac au Sud, Le Vigeant à l'Ouest, Thollet à l'Est. La majorité est située en "terres de brandes »; certaines, les plus au Sud, sur les «confins granitiques » sont plus favorisées avec des sols moins hydromorphes (figure 1).

\section{2 / Structures et moyens de production}

Les surfaces sont grandes (de 56 à 266 ha SAU, 106 ha de moyenne). Ces exploitations sont en majorité spécialisées en ovins viande. Seules, quatre possèdent aussi des vaches allaitantes limousines et une a également des chèvres.

La surface fourragère occupe $72 \%$ de la SAU en moyenne, avec de fortes variations; 6 ont plus de $90 \%$ de SFP et 8 sont relativement " céréalières " (28 à $45 \%$ de céréales et oléoprotéagineux dans la SAU). Les effectifs ovins sont importants (521 brebis en moyenne) : il s'agit de races d'herbage variées, choisies pour leur bonne conformation (la souche de base est souvent "vendéenne" avec utilisation de béliers Charollais, Suffolk, Texel). Un seul troupeau est de race Charmoise.

\section{3 / Fonctionnement des systèmes ovins}

Le système traditionnel était basé sur un agnelage de fin d'hiver avec une production d'agneaux finis à l'herbe.

Les éleveurs, en introduisant la culture des céréales, et encouragés par les prix de vente plus favorables de l'hiver et du printemps, se sont peu à peu tournés vers la production d'une partie de leurs agneaux en contre-saison (nés de septembre à janvier) qu'ils engraissent en bergerie avec des concentrés partiellement auto-produits. Simultanément le rythme d'agnelage a été accéléré en vue d'améliorer la productivité du troupeau, certaines brebis ayant deux mise bas dans l'exercice. En outre, afin d'accroître les surfaces consacrées aux céréales tout en conservant le même effectif ovin, les surfaces fourragères ont été intensifiées pour augmenter le chargement. Cette évolution s'est accompagnée d'une plus forte consommation d'intrants.

Economiquement valable il y a quelques années, ce système trouve aujourd'hui ses limites dans un contexte commercial très tendu où les prix de vente ne cessent de décroître et où le niveau des marges devient de plus en plus dépendant du montant des charges opérationnelles.

Les deux logiques de fonctionnement, système herbager " traditionnel " et système intensif se retrouvent dans notre échantillon avec une série de situations intermédiaires, correspondant à des phases de transition ou à des contextes spécifiques (très grandes structures, présence de salariés, présence de bovins, etc...).

Comment identifier ces différents types? Quels résultats obtiennent-ils?

\section{2 / Détermination des types ou profils d'exploitation}

\section{1 / Méthode}

Le but est de caractériser les différents modes de fonctionnement global des exploitations, en considérant la manière de conduire le troupeau en relation avec l'utilisation des surfaces fourragères, la structure et l'orientation générale de l'exploitation.

Pour tenir compte simultanément des différents indicateurs de fonctionnement, nous avons utilisé les méthodes d'analyses multi-critères associant une analyse en composante principale (ACP) et une classification ascendante hiérarchique (CAH) (Fénelon 1989), selon une démarche déjà utilisée avec nos collègues du CEMAGREF (Baud et al) dans l'analyse de nos réseaux concertés d'élevage bovin (CEMAGREF 1989).

Treize variables ont été finalement retenues pour caractériser :

- les structures (SAU, (nb UGB + 0,5 x ha céréales)/UTH, \% salariés, charges de structures comparatives/ha SAU),

le stade de développement, par le taux d'endettement,

- l'équilibre général du système (\% SFP, UGB ovins \% UGB totales),

- la conduite de l'atelier ovin (\% d'agneaux d'herbe commercialisés, marge brute par brebis),

- la réussite des cultures (marge par ha).

Trois indicateurs de revenus sont pris en variables supplémentaires (le revenu du travail et des capitaux propres d'exploitation, par ha SAU et par UTH, le Disponible I par UTH ${ }^{(2)}$.

\section{2 / Les facteurs de la dispersion}

Sept facteurs rendent compte de $87 \%$ de la variabilité globale, mais les deux premiers en expliquent à eux seuls $45 \%$. Le premier $(26 \%$ de la variabilité) correspond au degré de spécialisation fourragère et au niveau d'intensification (qui sont eux-mêmes liés). Le second ( $19 \%$ de la variabilité) renvoie aux structures d'exploitation en relation avec la conduite du troupeau ovin.

(2) Disponible $I=$ excédent brut d'exploitation + compte exceptionnel - Annuités d'emprunts (y compris foncier) + variations CT et Dettes - variations d'inventaire (des productions et des approvisionnements). Il correspond à ce qui reste pour les prélèvements privés, l'épargne et l'autofinancement des nouveaux équipements. 
Figure 2. Représentation de la classification hiérarchique (logiciel ADDAD). Méthode des voisins réductibles réalisée sur les 7 premiers facteurs, $n=25$.

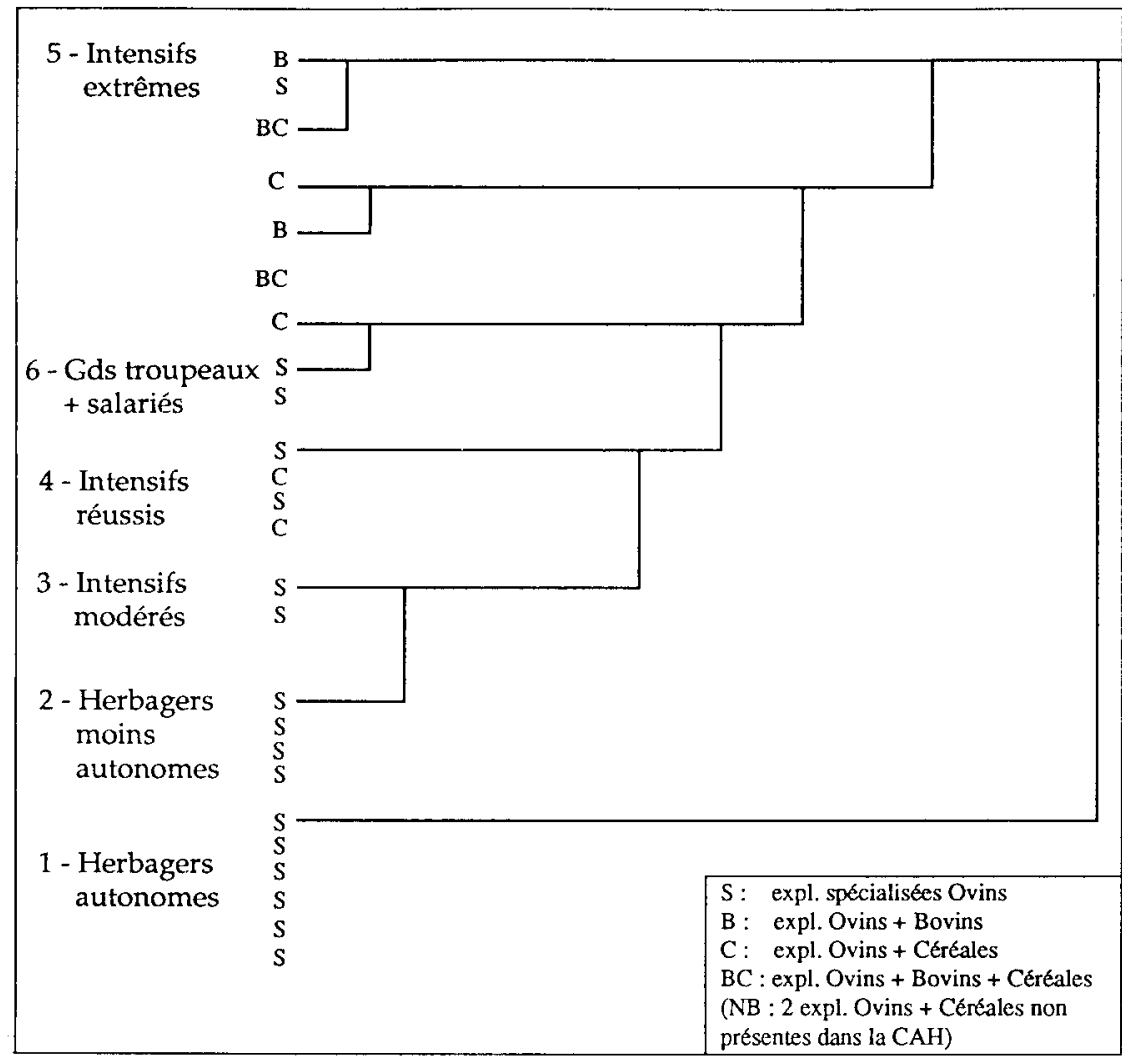

\section{3 / Détermination des profils}

- Six regroupements bien caractérisés et homogènes (ressortant de la $\mathrm{CAH}$ ) représentent différents modes de fonctionnement d'exploitations qui peuvent s'articuler autour de deux logiques contrastées mais cohérentes (figure 2) :

- celle d'une intensification fourragère assez forte, bien réussie, les «Intensifs Réussis » (IR)

- celle d'une exploitation plus extensive de la SFP, ayant conservé un mode de fonctionnement plus traditionnel, mais avec également de bons résultats, les "Herbagers Autonomes" (HA)

- autour de ces deux profils caractérisés, quatre autres sont également présents, et regroupent :

- des exploitations encore plus intensives que les IR, qualifiées, « d'Intensifs Extrêmes " (IE), - des exploitations ayant un niveau d'intensification intermédiaire entre les IR et les HA, constituées souvent d'entreprises non stabilisées et en développement, se séparant ellesmêmes selon le mode de conduite des surfaces et du troupeau en Intensifs Modérés (IM) et Herbagers moins autonomes $(\mathbf{H}-)$,

- des exploitations spécialisées ayant de grands troupeaux ovins, avec une main d'œuvre salariée.

- Un $7^{\circ}$ profil rassemble des exploitations plus hétérogènes mais dont la caractéristique commune est d'avoir une orientation céréalière plus marquée ( 28 à $45 \%$ de la SAU) et de dis- poser, en général, des plus grandes surfaces. Deux IR en font partie.

Une exploitation en évolution est restée hors profil.

\section{3 / Les différents profils d'exploitation}

L'analyse des trois systèmes les plus caractérisés, les Intensifs Réussis, les Herbagers Autonomes et les exploitations à tendance céréalière, permet de situer les enjeux, les choix possibles et les problèmes. En revanche, dans ce texte limité, on ne peut faire qu'une présentation très sommaire des autres profils, malgré leur intérêt, mais les résultats détaillés, qui figurent dans les tableaux joints, permettent au lecteur intéressé d'en faire l'analyse.

\section{1 / Les Intensifs Réussis}

$$
(n=4) \text { (tableau } 1)
$$

Il s'agit d'exploitations de moyenne dimension ( $86 \mathrm{ha}$ ) avec 1,9 UTH, presque entièrement familiales, $70 \%$ des terres sont en fermage. Ces éleveurs sont installés depuis au moins 10 ans. Le troupeau est presque exclusivement ovin. Deux exploitations disposent de bâtiments récents avec une distribution de l'alimentation entièrement mécanisée. Les deux autres ont aménagé et agrandi des bâtiments datant d'une trentaine d'années.

\section{a / La logique de fonctionnement}

Typiquement ils ont opté pour une logique d'intensification fourragère maximale avec un chargement relativement élevé : 1,52 UGB/ha SFP

Tableau 1. Les intensifs réussis.

86,5 ha de SAU

SFP / SAU : $73 \%$

540 brebis

UGB / UTH : 51

Capital d'exploitation : $12000 \mathrm{~F} /$ ha dont $43 \%$ en cheptel.

Taux d'endettement : $30 \%$.

Conduite technique

Chargement : 1,52 UGB / ha SFP

49 unités de $\mathrm{N} /$ ha SFP

$672 \mathrm{~F}$ de frais SFP / ha

Agneaux de bergerie : $67 \%$

Nombre d'agneaux produits par femelle : 1,43

Poids des agneaux : 17,8 kg net

Concentré : $190 \mathrm{~kg} / \mathrm{brebis}$

Autonomie fourragère : $63 \%$

Résultats économiques

Marge ovine finale : $542 \mathrm{~F}$ / brebis

Marge de la SFP : $4921 \mathrm{~F} / \mathrm{ha}$ $164000 \mathrm{~F} / \mathrm{UTH}$

Marge des cultures : $3040 \mathrm{~F} /$ ha $38300 \mathrm{~F} / \mathrm{UTH}$

Charges de structure comparatives : $2418 \mathrm{~F}$ / ha SAU

Revenu du travail et des capitaux :

$2147 \mathrm{~F} / \mathrm{haSAU} \quad 98000 \mathrm{~F} / \mathrm{UTH}$ 
(pour l'ensemble brebis + agneaux), dont $45 \%$ sont autoproduits et $55 \%$ achetés.

Néanmoins ces éleveurs ont aussi le souci de leur autonomie fourragère : $42 \%$ de la SFP servent aux stocks fourragers, avec une place importante réservée à l'ensilage $20 \%$ de la SFP), tant en herbe qu'en maïs (dont les $3 / 4$ vont aux ovins). On fait aussi des cultures dérobées (colza et autre) : 1,5 ha par exploitation. La fertilisation minérale est relativement forte (49 25 - 36 NPK/ha SFP) et le total des frais de production fourragère atteint $672 \mathrm{~F} /$ ha SFP.

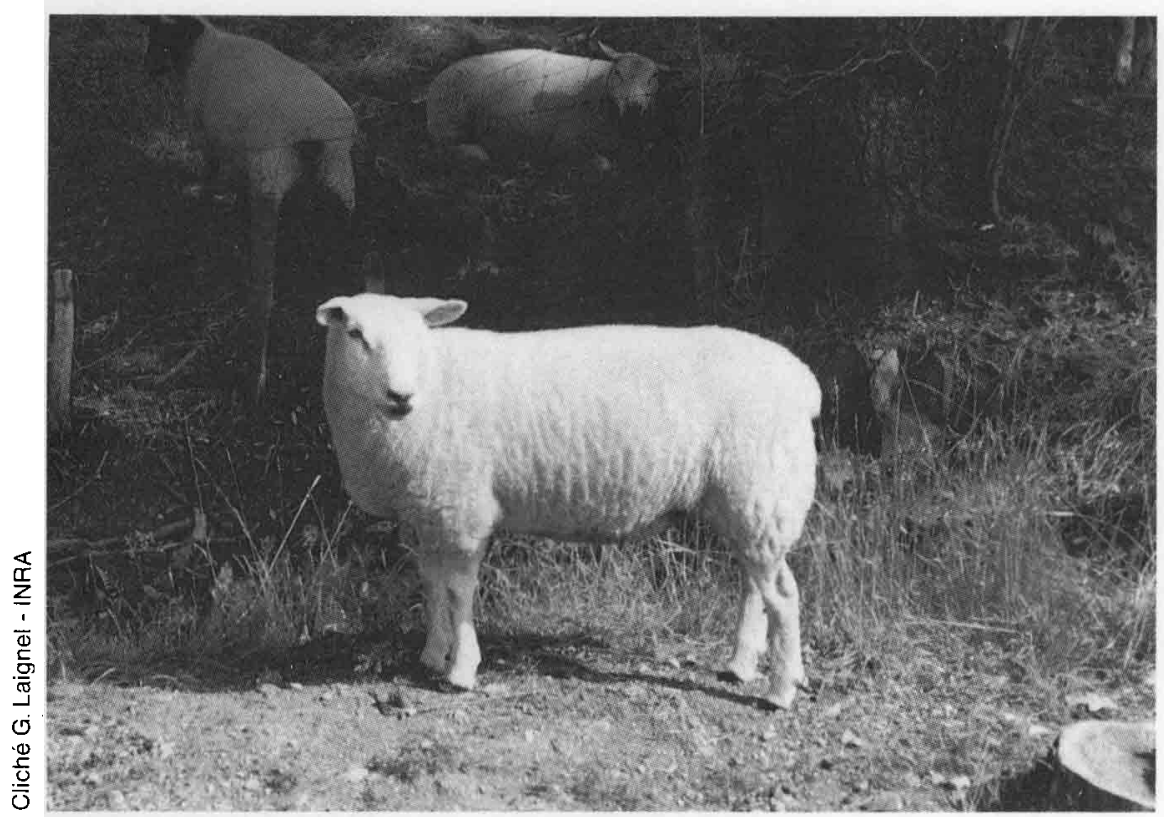

Type génétique fréquent, la brebis Texel est appréciée pour son format et son adaptation au pâturage.

\section{b / Les résultats ovins}

La productivité du troupeau est bonne, 1,43 agneaux produits par brebis (femelle de plus de 12 mois), grâce à un taux d'agnelage par brebis assez élevé $(112 \%)$ et malgré une mortalité un peu forte $(13 \%)$. Les deux tiers des agneaux sont menés en bergerie, et $12 \%$ seulement sont finis exclusivement à l'herbe (sans concentré ou sans finition à l'intérieur). Le poids de carcasse est correct $(17,8 \mathrm{~kg})$ et le prix moyen de vente atteint $540 \mathrm{~F}$ par agneau (1989).

Le produit par brebis est élevé (951 F). Les charges le sont aussi, avec $338 \mathrm{~F}$ relatives au troupeau (dont $257 \mathrm{~F}$ de concentré) et $71 \mathrm{~F}$ pour la production fourragère. Elles absorbent ainsi $43 \%$ du produit. Néanmoins la marge obtenue est de bon niveau avec $542 \mathrm{~F}$ par brebis.

\section{c / Les résultats globaux}

La marge par hectare SFP atteint $4920 \mathrm{~F}$, ce qui est élevé, contre $3040 \mathrm{~F}$ pour les cultures (77\% en céréales à 52 qx/ha, 1989 étant une bonne année). La marge globale (aides comprises) s'élève à $4640 \mathrm{~F}$ par ha SAU et $213000 \mathrm{~F}$ par travailleur.

1 La contrepartie de l'intensification fourragère est la nécessité d'un capital relativement important: $12000 \mathrm{~F} /$ ha et $551000 \mathrm{~F}$ par travailleur, dont plus de la moitié est due aux équipements, matériels et bâtiments, qui alourdissent les charges de structure $(657 \mathrm{~F}$ d'amortissement par ha SAU). Plus généralement, le poste le plus important des charges de structure est la mécanisation $(760 \mathrm{~F} / \mathrm{ha})$ mais les charges foncières arrivent en deuxième position $(700 \mathrm{~F}$ de charges réelles et $840 \mathrm{~F}$ si on tient compte de la valeur locative des terres en propriété). Fort heureusement, le taux d'endettement de ces exploitations n'est pas trop élevé $(35 \%$ en début) et les frais financiers, avec $226 \mathrm{~F} / \mathrm{ha}$ ne sont pas excessifs, comparativement du moins à ce qu'on rencontre dans les autres profils. Au total les charges de structure (plus les charges proportionnelles non affectables) atteignent $2416 \mathrm{~F} / \mathrm{ha}$, soit plus de la moitié de la marge.

$\mathrm{Au}$ total, le revenu (revenu agricole ou résultat courant) dépasse $2200 \mathrm{~F} / \mathrm{ha}$. En tenant compte des terres en propriété (et de la petite proportion de travail salarié), le revenu du travail et capitaux propres est de $2147 \mathrm{~F} /$ ha soit près de $100000 \mathrm{~F} /$ travailleur. Résultat que l'on peut considérer comme largement supérieur à la moyenne des éleveurs ovins français (Chaalali 1990).

Ces exploitations ont poursuivi en 1989 un fort développement puisqu'elles ont à la fois réduit leur endettement d'exploitation (de 35 à $30 \%$ ), fait face à des annuités foncières lourdes, en ayant beaucoup investi en équipement $(106000 \mathrm{~F}$, dont $85 \%$ par autofinancement). Dans ces conditions le disponible (II) ${ }^{(3)}$ qui reste pour la famille et l'épargne est très réduit (34000 F par travailleur familial).

En définitive, le revenu d'exploitation est de bon niveau; il est obtenu sans doute avec une charge de travail élevée (285 brebis, 51 UGB dont quelques bovins et chèvres, et 12 ha de céréales, par travailleur), grâce à une excellente technicité mais avec beaucoup d'investissements à faire qui réduisent le disponible restant pour la famille.

\section{2 / Les Herbagers Autonomes}

$$
(n=6) \text { (tableau 2) }
$$

Ce profil est très homogène et très distinct des autres. Dans la classification hiérarchique ascendante, il est le premier à se différencier de tous les autres profils (figure 2).

\section{a / La logique de fonctionnement}

Il s'agit d'exploitations de surfaces comparables à celles des Intensifs Réussis (87 ha), avec un peu moins de main-d'œuvre $(1,6 \mathrm{UTH})$ et davantage de terre en propriété (70\% contre $30 \%$ ). Mais la logique est très différente. La spécialisation ovine est très prononcée: 500 brebis, aucun bovin et surtout très peu de cultures ( $5 \%$ ). Ici on n'a pas cherché à intensifier la surface fourragère pour dégager des surfaces au profit des cultures. Ce qui ne signifie pas que l'herbe ne soit pas bien gérée, mais on a opté pour un chargement modeste $(1,02$ UGB/ ha), avec des frais réduits. Très peu de parcelles

(3) Disponible $I I$ - Disponible I - Achats d'équipements (matériel, bâtinents, améliorations foncières) + Nouveaux emprunts reçus (sauf ceux pour les reprises) 


\section{Tableau 2. Les herbagers autonomes}

\section{7,4 ha de SAU \\ SFP / SAU : $95 \%$ \\ 495 brebis}

UGB / UTH : 52

Capital d'exploitation : $7700 \mathrm{~F} /$ ha dont $52 \%$ en cheptel.

Taux d'endettement : $34 \%$.

\section{Conduite technique}

Chargement : 1,02 UGB / ha SFP

7 unités de N / ha SFP

$259 \mathrm{~F}$ de frais SFP / ha

Agneaux de bergerie : $25 \%$

Nombre d'agneaux produits par femelle : 1,42

Poids des agneaux : $18,5 \mathrm{~kg}$ net

Concentré : $107 \mathrm{~kg} / \mathrm{brebis}$

Autonomie fourragère : $75 \%$

\section{Résultats économiques}

Marge ovine finale : $670 \mathrm{~F}$ / brebis

Marge de la SFP : $4039 \mathrm{~F} / \mathrm{ha}$ $208000 \mathrm{~F} / \mathrm{UTH}$

Marge des cultures : $2695 \mathrm{~F} / \mathrm{ha}$

$$
7020 \mathrm{~F} / \mathrm{UTH}
$$

Charges de structure comparatives :

$1983 \mathrm{~F} /$ ha SAU

Revenu du travail et des capitaux :

$2119 \mathrm{~F} / \mathrm{ha}$ SAU $\quad 115000 \mathrm{~F} / \mathrm{UTH}$

sont drainées ( $3 \%$ de la SAU), mais les sols en ont peut-être un peu moins besoin, car la plupart sont situés au Sud de la Vienne ou Nord de la Haute-Vienne sur «les confins granitiques ». Le mode d'utilisation choisi pour les sols n'exige pas non plus le drainage. Ces exploitants sont d'abord des « moutonniers".

\section{b / Utilisation des surfaces fourragères}

Malgré le choix d'un certain extensif, l'herbe est cultivée, car il n'y a que $9 \%$ de prairies permanentes. La plupart des prairies sont régulièrement resemées tous les 5 à 6 ans et implantées avec des mélanges complexes, à base de ray-grass italien, trèfle blanc, parfois trèfle violet, ray-grass anglais, fétuque, lotier, etc. La première année (voire la deuxième) la jeune prairie, où dominent RGI et TV, est pâturée par les agneaux : outre la qualité de l'herbe, cela a l'avantage de réduire le parasitisme et aussi le piétinement. Les brebis exploitent les années suivantes. Parfois les semis sont destinés à une rotation plus rapide (avec RGI et TV). Ils sont également destinés aux agneaux à l'engraissement, et ils sont mis en place pour faire face à un déficit fourrager et assurer ainsi la sécurité du système.

La fertilisation minérale est modérée et conçue pour favoriser le trèfle (7 - 27 - 24 NPK) ha SFP). En outre la récolte des stocks est presque entièrement à base de foin (un seul éleveur fait de l'ensilage d'herbe). On arrive ainsi à une dépense de production fourragère réduite à 259 F/ha SFP.

\section{c / Conduite du troupeau ovin}

Elle est fondée sur un agnelage principal de printemps qui représente les $3 / 4$ des mise bas annuelles. Cinq éleveurs sur six pratiquent un

rattrapage d'automne. Trois sur six accélèrent un peu les agnelages, avec deux mise bas pour une partie des brebis, souvent celles de réforme. L'accent est mis sur un suivi très précis des brebis improductives. Les éleveurs obtiennent ainsi 1,10 agnelage/femelle de plus de 12 mois, conséquence de ce suivi, et du mode de calcul du ratio qui prend en compte les durées de présence et donc la plus ou moins bonne gestion des réformes. La productivité atteint un bon niveau, 142 agneaux produits pour 100 femelles de plus de 12 mois.

Les agneaux nés au printemps sont engrais sés au maximum à l'herbe, et vendus de fin juin à décembre voire janvier. Les cours défavorables durant cette période sont compensés par des poids de carcasse élevés. Les agneaux de contre-saison nés de fin octobre à janvier sont, selon les lots et les conditions du prin-

\section{Herbagers autonomes}

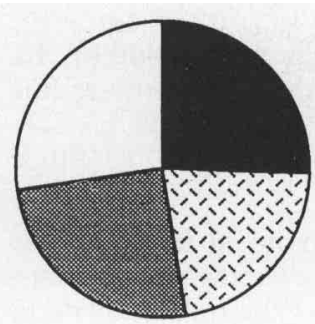

Intensifs modérés

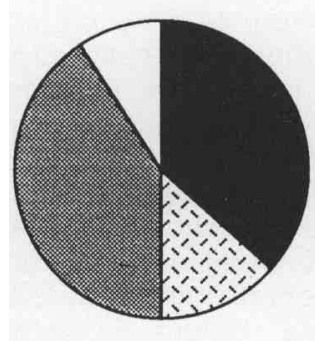

Intensifs extrêmes

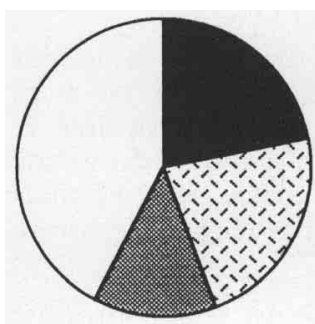

Grandes SAU + cultures

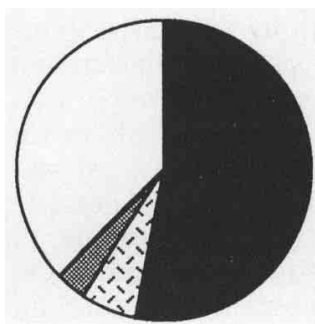

Herbagers moins autonomes

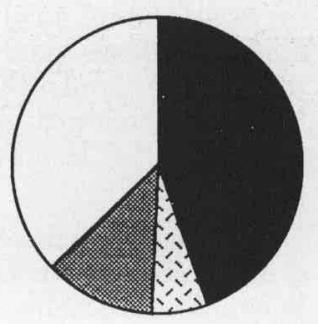

Intensifs réussis

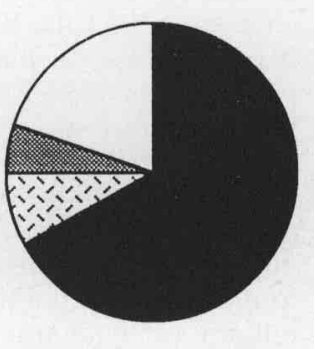

Grands troupeaux + salariés

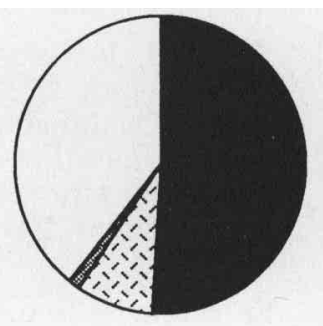

agneaux de bergerie Eagneaux d'herbe

agneaux herbe + concentrés agneaux d'herbe finis en bergerie
Figure 4. Répartition des ventes d'agneaux selon les catégories, par profil d'exploitation. 
temps, engraissés entièrement en bergerie ou finis à l'herbe en avril-mai. Au total, $75 \%$ des agneaux sont menés à l'herbe, dont $20 \%$ sont entièrement finis sans concentré, et $25 \%$ sont engraissés entièrement en bergerie (figure 4). Le poids moyen $(18,5 \mathrm{~kg}$ de carcasse) et le prix ( $556 \mathrm{~F}$ pièce) obtenus sont les plus élevés de tous les profils.

La productivité pondérale exprimée en kilo de carcasse d'agneaux atteint $25,1 \mathrm{~kg} / \mathrm{brebis}$, contre $24,3 \mathrm{~kg}$ chez les intensifs réussis (IR). Cette performance est obtenue avec beaucoup moins de concentré, $107 \mathrm{~kg} / \mathrm{brebis}$ (dont $80 \%$ sont achetés). La productivité autonome atteint $18,9 \mathrm{~kg}$ d'équivalent carcasse, contre $15,3 \mathrm{~kg}$ pour les IR, soit une autonomie fourragère de $75 \%$, contre $63 \%$.

Le produit par brebis est comparable à celui des IR (959 F), mais les charges n'en absorbent que $30 \%$, et la marge est beaucoup plus élevée : $670 \mathrm{~F} / \mathrm{brebis}$.

\section{d / Résultats généraux}

Si la marge par hectare de SFP est naturellement inférieure à celle des IR ( $4039 \mathrm{~F} /$ ha SFP), elle la dépasse par travailleur (208000 F). La très faible contribution des cultures réduit l'écart des marges globales, $4141 \mathrm{~F} / \mathrm{ha}$ SAU et $224000 \mathrm{~F} / \mathrm{UTH}$, contre $4639 \mathrm{~F}$ et $213000 \mathrm{~F}$ pour les IR

Mais la spécialisation ovine et le parti pris d'une faible intensification fourragère limitent le capital nécessaire : $7700 \mathrm{~F} / \mathrm{ha}$ dont plus de la moitié sous forme de cheptel, et $417000 \mathrm{~F} / \mathrm{tra}-$ vailleur, soit $25 \%$ de moins que chez les IR. Les charges de structure sont, de ce fait, moins élevées, $1709 \mathrm{~F} /$ ha SAU (contre $2416 \mathrm{~F}$ pour les IR). Les frais de mécanisation sont beaucoup plus faibles (394 F), tout comme les charges foncières qui ne comportent pas de frais de drainage ( $1 \mathrm{~F}$ contre $100 \mathrm{~F} / \mathrm{ha}$ ). En revanche, les frais de bâtiments sont dans la moyenne, quatre éleveurs n'utilisent que des vieux bâtiments mais il y a l'entretien, deux autres ont construit une bergerie-appentis en complément. L'endettement est également limité : le taux de $34 \%$ pour un capital plus faible signifie moins d'encours à l'hectare et donc moins de frais fínanciers (148 F contre $226 \mathrm{~F}$ pour les IR).

$\mathrm{Au}$ total, le résultat courant est supérieur (2432 F contre $2232 \mathrm{~F} / \mathrm{ha}$ SAU). Si on tient compte de la différence de terre en propriété, le revenu du travail et des capitaux est du même ordre à l'hectare (2119 F contre $2147 \mathrm{~F}$ ) mais plus élevé par travailleur: $114600 \mathrm{~F}$, contre $98400 \mathrm{~F}$.

En revanche la trésorerie est beaucoup plus favorable que celle des IR. S'il y a d'assez lourdes annuités foncières dans les deux cas, les remboursements en capital d'emprunt d'exploitation sont ici plus réduits : ils correspondent d'ailleurs au montant des amortissements eux-mêmes moins élevés. Ces éleveurs sont légèrement plus âgés que les précédents et un peu plus « en croisière ». Le troupeau est stable, les nouveaux investissements en équipements correspondent aux amortissements et sont faits à $30 \%$ par autofinancement. On maintient clairement l'appareil de production, en le renouvelant, tout en diminuant l'endettement. Le disponible II (prélèvements privés + épargne) est ainsi nettement supérieur à tous les autres profils (94000 $\mathrm{F}$ par travailleur familial).

En définitive l'écart de trésorerie et de disponible II s'explique en partie par une dynamique différente provenant d'un stade de développement différent. La situation d'aujourd'hui traduit un bon fonctionnement de ces exploitations depuis plusieurs années (les résultats 1988 étaient déjà du même ordre).

Si on se réfère à l'efficacité économique globale mesurée par le résultat courant, l'écart par rapport aux IR peut certes apparaître assez faible. Mais elle est obtenue avec beaucoup moins de capital. Surtout, il semble qu'elle soit acquise avec moins de travail, même si cela est bien difficile à quantifier. Certes, l'importance du cheptel est comparable ( 52 UGB/UTH, contre 51), mais le système est beaucoup plus simple chez les Herbagers Autonomes, très peu de cultures, conduite des brebis moins contraignante en moyenne sur l'année, peu de pose d'éponges ( $7 \mathrm{~F}$ contre $13 \mathrm{~F}$ ), moins d'agnelages en contresaison (figure 3), moins d'agneaux engraissés en bergerie. Elément souvent apparu, non chiffrable mais bien significatif, les éleveurs de ce profil apparaissent beaucoup plus disponibles, en dehors naturellement de la période d'agnelage de février à avril.

Enfin, cet équilibre est sans doute moins vulnérable à la dégradation de la conjoncture que celui des IR si on ajoute à la diminution du prix des ovins celle du prix des cultures, qui se cumule avec des fluctuations de rendements relativement amples.

Tableau 3. Les intensifs extrêmes.

75 ha de SAU
SFP / SAU : $75 \%$
443 brebis
UGB / UTH : 41
Capital d'exploitation : $16000 \mathrm{~F}$ / ha dont $38 \%$
en cheptel.
Taux d'endettement : $85 \%$.
Conduite technique
Chargement : 1,63 UGB / ha SFP
78 unités de N / ha SFP
$963 \mathrm{~F}$ de frais SFP / ha
Agneaux de bergerie : $21 \%$
Nombre d'agneaux produits par femelle : 1,31
Poids des agneaux : $18 \mathrm{~kg}$ net
Concentré : $185 \mathrm{~kg} / \mathrm{brebis}$
Autonomie fourragère : $57 \%$
Résultats économiques
Marge ovine finale : $444 \mathrm{~F} / \mathrm{brebis}$
Marge de la SFP : $4132 \mathrm{~F} / \mathrm{ha}$
$\quad 104000 \mathrm{~F} / \mathrm{UTH}$
Marge des cultures : $2356 \mathrm{~F}$ / ha
Charges de structure comparatives :
$3665 \mathrm{~F}$ / ha SAU
Revenu du travail et des capitaux :
$367 \mathrm{~F}$ / ha SAU $12000 \mathrm{~F}$ / UTH




\section{3 / Les Intensifs Extrêmes}

$$
\text { (n = 2) (tableau 3) }
$$

Ces exploitations sont limitées en surface (75 ha pour 2,25 travailleurs). Et l'intensification apparaît pour l'instant comme la seule voie possible. $78 \%$ de la SAU ont été drainés.

La logique de fonctionnement est proche de celle des IR, mais ses caractères en sont accentués. Le chargement est plus élevé encore $(1,63)$. Cela permet de concilier les cultures $(25 \%)$ et un troupeau d'effectif assez important (443 brebis, avec pour une exploitation 18 vaches allaitantes). Mais la maîtrise technique est mal assurée, la mortalité élevée des agneaux en est un signe. Les charges opérationnelles, tant pour la production fourragère que pour le troupeau, sont trop lourdes par rapport au produit obtenu, et la marge par brebis est réduite. Les charges de structure, particulièrement celles de mécanisation et de financement sont élevées, car le capital et les emprunts sont importants. Dès lors, le revenu est faible et la trésorerie inexistante, l'endettement s'accroît, conséquences de la surface limitée et plus encore de l'insuffisance d'efficacité économique.

On est ici en présence de situations particulièrement difficiles. Ces exploitations, surendettées, n'arrivent pas à conduire de pair une intensification fourragère très poussée et une bonne conduite du troupeau avec une limitation des coûts d'alimentation. L'autonomie fourragère est très faible $(57 \%)$. L'intensification poussée qui entraîne des coûts et des investissements lourds n'arrive plus à générer un produit suffisant. Peut-être est-on allé audelà des limites permises par le milieu pédoclimatique difficile dans lequel sont situées ces exploitations?

\section{4 / Deux profils intermédiaires :}

- Les herbagers moins autonomes $(n=4)$ (tableau 4)

- Les Intensifs Modérés ( $n=2)$ (tableau 5)

Il s'agit dans les deux cas d'exploitations de surface moyenne ( 82 et 78 ha) avec un peu moins de main d'cuvre que les profils précédents, notamment les intensifs modérés $(1,30$ UTH). Les installations sont relativement récentes (entre 3 et 12 ans), le système n'est pas stabilisé et l'endettement est élevé $(58 \%$ chez les Herbagers moins autonomes, $86 \%$ chez les Intensifs Modérés). Les remboursements sont importants, avec fort heureusement des annuités foncières plus faibles.

Les troupeaux ovins sont de même dimension ( 435 brebis), il n'y a pas de bovins, et les $20 \%$ de cultures servent en grande partie à l'alimentation du troupeau. Les éleveurs n'ont pas encore acquis toute la maîtrise technique nécessaire.

Chez les Herbagers moins autonomes, l'intensification fourragère n'est pas très poussée mais les dépenses sont cohérentes avec le chargement actuel. Les points faibles se situent dans la conduite du troupeau dont les résultats sont les plus faibles de tous malgré les efforts
Tableau 4. Les herbagers moins autonomes.

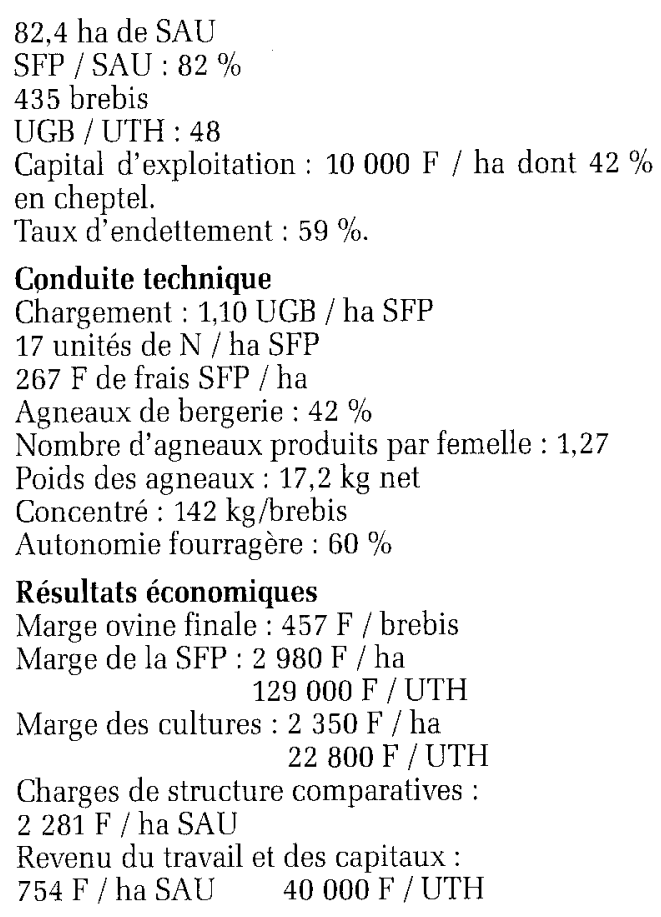

\section{Tableau 5. Les intensifs modérés.}

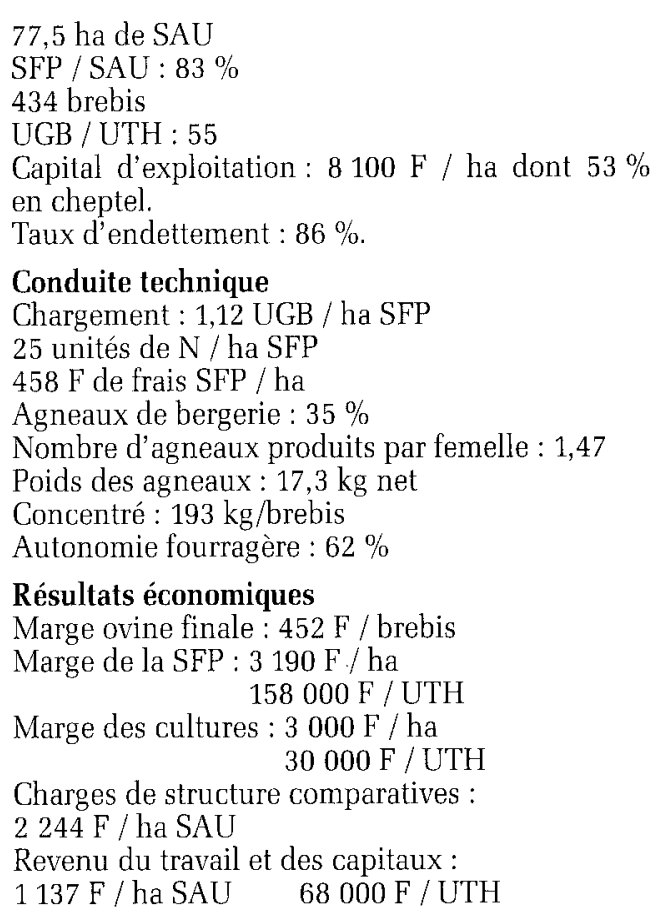

faits (accélération, contre-saisons, etc). Néanmoins les éleveurs en sont conscients, deux s'engagent dans un suivi individuel de leurs brebis.

Les Intensifs Modérés obtiennent de meilleurs résultats zootechniques, mais ce sont les charges qui apparaissent mal proportionnées 
au produit, notamment le concentré, entraînant une faible autonomie fourragère. En outre le bon équilibre entre les dépenses de production fourragère et le chargement possible reste encore assez difficile à trouver.

\section{5 / Grands troupeaux avec salariés : production d'agneaux de bergerie $(n=2)$ (tableau 6)}

Dans ces exploitations de grande dimension (114 ha, 755 brebis), la main d'œuvre, en partie salariée, est limitée. Le système choisi est relativement simplifié : uniquement des brebis, peu de cultures.

L'option est de finir la majorité des agneaux en bergerie, pratique plus facile à gérer que l'engraissement à l'herbe. Pour cela, une partie des agnelages se fait à l'automne. Malheureusement la productivité du troupeau reste moyenne, car les grands effectifs rendent difficile une élimination régulière des brebis improductives. Mais la principale caractéristique est l'importance des charges opérationnelles, spécialement le concentré, qui absorbent la moitié du produit. La marge par brebis est faible. Et les charges de structure, très élevées, réduisent le revenu par travailleur ( $50000 \mathrm{~F}$, la moitié de celui des IR et des HA).

En définitive, l'importance des effectifs mis en jeu ne permet pas de rattraper le bas niveau de la marge par brebis.

Visiblement la productivité économique du troupeau est insuffisante. Est-ce dû au grand nombre de brebis qui en limite les possibilités de gestion? Est-ce dû à la main d'œuvre salariée elle-même, malgré une adaptation opportune du système avec un souci de simplification et de rationalisation? Mais celle-ci génère

Tableau 6. Grands troupeaux + salariés.

114 ha de SAU
SFP / SAU : $86 \%$
755 brebis
UGB / UTH : 79
Capital d'exploitation : $8800 \mathrm{~F}$ / ha dont $57 \%$
en cheptel.
Taux d'endettement : $79 \%$.
Conduite technique
Chargement : 1,32 UGB / ha SFP
40 unités de N / ha SFP
$683 \mathrm{~F}$ de frais SFP / ha
Agneaux de bergerie : $51 \%$
Nombre d'agneaux produits par femelle : 1,36
Poids des agneaux : $16,7 \mathrm{~kg}$ net
Concentré : $162 \mathrm{~kg} / \mathrm{brebis}$
Autonomie fourragère : $54 \%$
Résultats économiques
Marge ovine finale : $420 \mathrm{~F}$ / brebis
Marge de la SFP : $3233 \mathrm{~F} / \mathrm{ha}$
$\quad 195000 \mathrm{~F} / \mathrm{UTH}$
Marge des cultures : $1554 \mathrm{~F}$ / ha
$\quad 15250 \mathrm{~F} / \mathrm{UTH}$
Charges de structure comparatives :
$2307 \mathrm{~F} /$ ha SAU
Revenu du travail et des capitaux :
$716 \mathrm{~F}$ / ha SAU $50000 \mathrm{~F}$ / UTH

des coûts supplémentaires en intrants, que la diminution constante des prix de vente va rendre de moins en moins supportables.

\section{6 / Les grandes exploitations à tendance céréalière \\ $(\mathrm{n}=8)$ (tableau 7)}

Il s'agit de grandes exploitations à fermage dominant. Les cultures de vente ne sont pas prédominantes mais elles sont assez développées (33\% de la SAU). La main d'œuvre est guère supérieure à la moyenne mais elle comporte des salariés. Les effectifs ovins sont importants (602 brebis) avec quelques bovins, au total près de 110 UGB. Elles se caractérisent par:

Une intensification fourragère modérée avec un chargement moyen $[1,16)$ : la main d'œuvre limite les effectifs de troupeau et les cultures accaparent une partie du travail.

- Des résultats du troupeau un peu faibles: la productivité est réduite (124\%) par la mortalité et les brebis conservées improductives. En outre, la moitié des agneaux est engraissée en bergerie. Au total, la productivité pondérale est plutôt modeste (19 kg), avec des charges trop fortes qui conduisent à une marge inférieure de $10 \%$ à la moyenne, que compensent heureusement, en partie, les effectifs.

- Des cultures qui ont donné de bons résultats en 1989, surtout le tournesol.

- Des résultats globaux amoindris par les charges. En effet les charges de structure sont élevées malgré la surface, notamment le matériel lié aux cultures. Au total le revenu par travailleur est heureusement supérieur à la moyenne, mais il ne dépasse pas ceux des HA et des IR.

\section{Tableau 7. Grandes SAU avec cultures.}

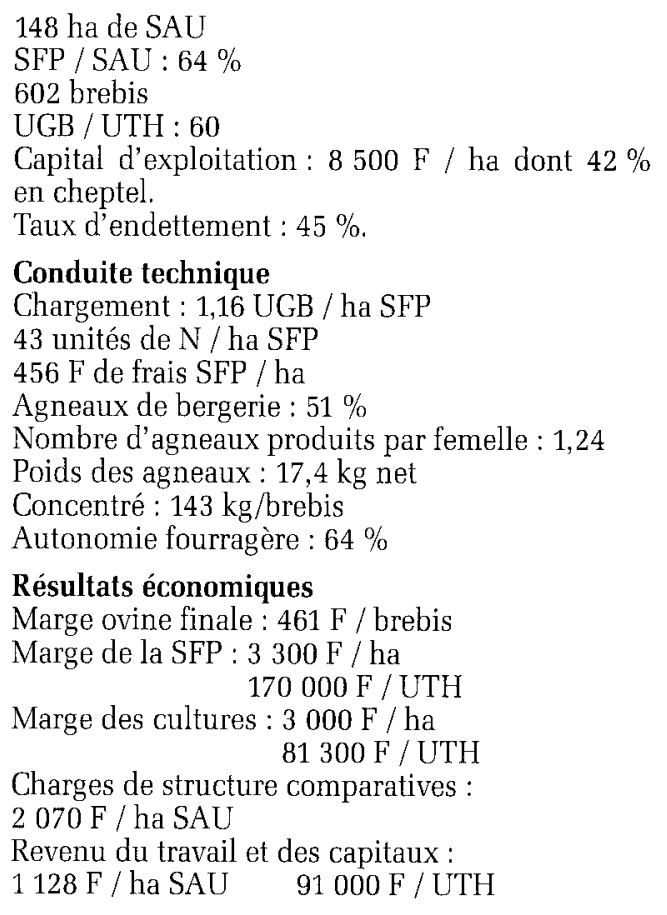


En août 1991, malgré la sécheresse, les agneaux sont engraissés à l'herbe sur des prairies nouvellement semées.

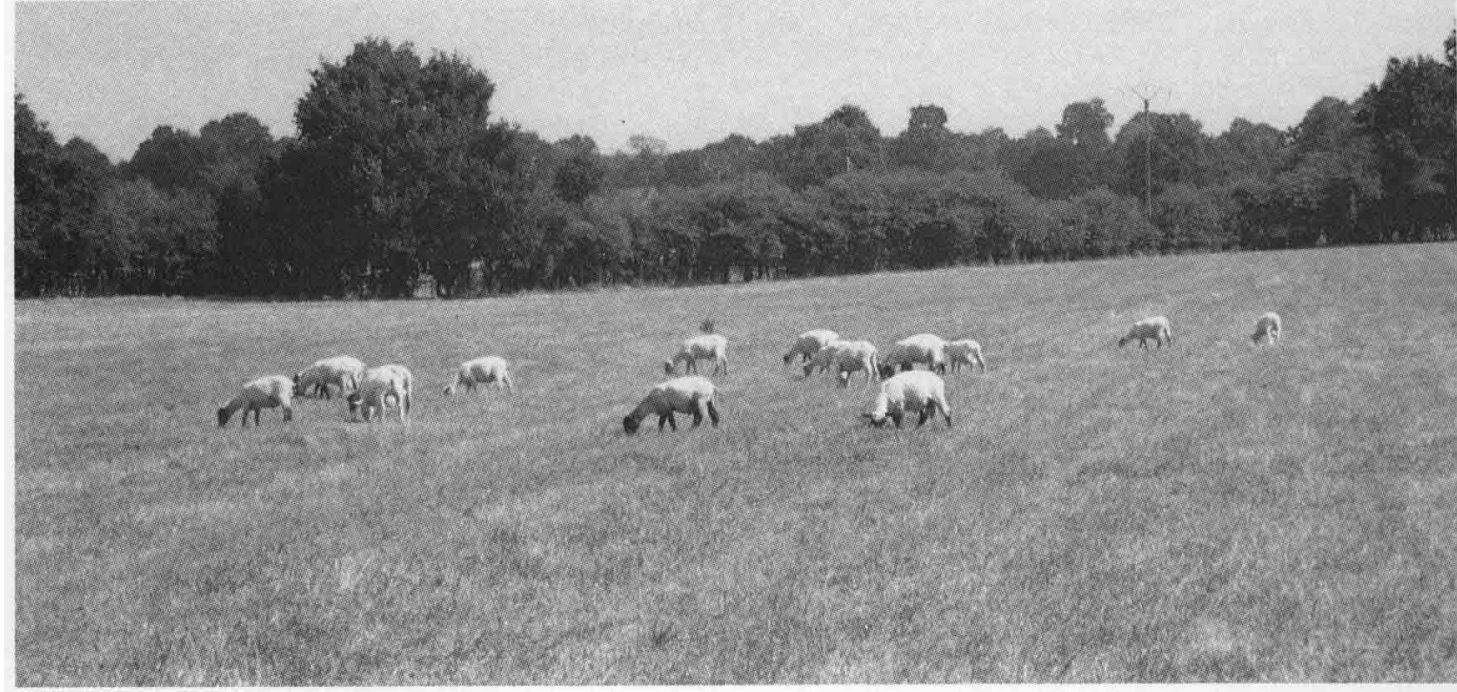

chargement, on peut ainsi exploiter une troupe d'effectifs suffisants en lui associant une sécurité alimentaire complémentaire procurée par les céréales (et la paille), tout en diversifiant les ventes. Cette voie est exigeante en technicité, travail et investissements. L'exemple des Intensifs Réussis montre clairement que pour dégager un revenu suffisant, il faut que l'intensification soit, maintenant, parfaitement raisonnée. C'est-à-dire, entre autres, que l'éleveur résiste aux facilités qu'entraîne avec lui ce système, notamment l'usage trop libéral des intrants, dont l'excès était tout à fait permis lorsque la conjoncture était meilleure, lorsque le rapport des prix entre les ovins, les céréales et les facteurs de production étaient plus favorables aux ovins. Il est sans doute aussi nécessaire que les éleveurs identifient mieux les limites à ne pas dépasser dans un milieu où les contraintes pédoclimatiques sont indéniables. Le travail engagé par nos collègues agronomes contribuera à préciser ce point important.

L'autre voie, suivie par les Herbagers Autonomes, peut davantage surprendre, puisqu'elle semble plus traditionnelle. Elle peut apparaître aujourd'hui comme une autre réponse possible aux contraintes actuelles. La bonne santé financière de la majorité de ces éleveurs qui ont conservé cette voie, montre qu'elle n'était pas non plus inadaptée dans le passé récent. L'équilibre est tout autre: le moindre chargement est compensé par une spécialisation fourragère et ovine presque complète, car l'objectif est là aussi de conduire un troupeau d'effectifs suffisants. La simplification générale du système permet d'obtenir une bonne productivité du troupeau auquel on peut se consacrer presque à plein temps aux périodes critiques. Ainsi, s'ils n'enregistrent pas encore la carrière de leurs brebis, leur carnet d'agnelage annuel leur permet un suivi rigoureux avec élimination systématique des improductives. Néanmoins, la moindre intensification fourragère ne signifie pas que ces moutonniers soient dépourvus d'une bonne gestion de leurs fourrages. Au contraire, ces éleveurs nous ont révélé un grand savoir-faire dans l'utilisation de leurs prairies, dont ils savent préserver la qualité (notamment la présence de légumineuses), sans pour autant sacrifier la productivité du troupeau. La spécialisation du système leur permet d'investir davantage en observations et en pratiques fourragères, qui ne se révèlent pas moins complexes que celles des intensifs. Le résultat est une bonne productivité globale du travail, près de $7700 \mathrm{~kg}$ d'équivalent carcasse produits par travailleur en viande brute, et près de $5800 \mathrm{~kg}$ en viande autonome. Chiffres sans doute à retenir. Pourtant des questions se posent aussi pour eux. Le débouché pour les agneaux lourds de qualité se maintiendra-t-il dans l'avenir? Ne faudrait-il pas désaisonner un peu plus pour se situer davantage en période favorable de vente (dont les extrêmes se creusent), comme le souhaite la filière ovine... et aussi pour mieux faire face aux sécheresses estivales? Mais ceci exigera davantage de stocks et de céréales... La dégradation continue des prix de ventes qui a une forte incidence chez ces éleveurs productifs ${ }^{(4)}$ fait inévitablement ressurgir la question d'une diversification des recettes..

Pour les grandes exploitations avec salariés, dont l'importance est indéniable dans l'occupation de ce territoire, les choix sont plus difficiles encore. Les charges de structure et l'endettement sont là, notamment les fermages qui ont permis l'agrandissement, les salaires et les charges sociales dont les montants s'alourdissent différentiellement, le coût des équipements déjà réalisés... La « diversification culturale » permet d'utiliser le territoire sans trop accroître un troupeau dont l'effectif déjà conséquent pose effectivement des problèmes de gestion. L'adoption des oléoprotéagineux pouvait représenter une bonne solution. Malheureusement, dans les situations de sol un peu limites, comme celles où se trouvent les exploitations suivies, l'irrégularité des rendements est forte ; l'insécurité des prix née des nouvelles règles de la PAC réduit beaucoup l'intérêt économique de ces productions dont les marges deviennent extrêmement fluctuantes. Ainsi, en 1990, si les marges céréalières se sont maintenues au même niveau qu'en 1989 (2377 F/ha en moyenne), celles des autres cultures de vente

(4) Une baisse de $3 \mathrm{~F} / \mathrm{kilo}$ de carcasse d'agneau fait baisser, toutes choses égales par ailleurs, le revenu de $23000 \mathrm{~F} / \mathrm{tra}$ vailleur soit près d'un quart... (et le revernu devient nul chez les Intensifs Extrêmes). 
ont diminué de $1650 \mathrm{~F}$ par ha $(-40 \%)$. Faut-il accroitre à nouveau le troupeau? Cela ne va pas dans le sens des éleveurs qui ont tendance à réduire la main d'œuvre salariée et donc le troupeau qui est le plus exigeant en travail. L'étude montre aussi les limites qu'entraînent, dans l'état actuel des techniques et des modes de conduite, les très grands effectifs, dont la gestion par lots importants ne peut plus être aussi fine, la surveillance aussi grande, surtout s'il y a un secteur de cultures de vente qui devient fatalement prioritaire. L'exemple des «grands troupeaux en bergerie» (où il y a moins de cultures) est significatif du problème : une production par travailleur très élevée, $9500 \mathrm{~kg}$ d'équivalent carcasse agneaux par UTH, en brut, se réduit à $5155 \mathrm{~kg}$ autonomes, soit moins que les Herbagers Autonomes qui ont des troupeaux de plus faibles effectifs et obtiennent un revenu plus élevé. En outre l'augmentation du nombre supposerait de nouveaux bâtiments, perspective peu encourageante dans la conjoncture actuelle. Faudrait-il revenir au plein air? Certains ne l'ont pas entièrement abandonné puisqu'ils réservent leur bergerie, par rotation, aux lots en agnelage pendant 3 à 4 semaines; et le système n'est pas dépourvu d'inconvénients avec les ovins... Finalement, n'est-on pas dans une situation où le gel d'une partie des terres en culture, avec ou sans jachère pâturée, pourrait être intéressant? La possibilité de faire des jachères annuelles risque d'en accroître l'attractivité, en particulier vis-à-vis des céréales de printemps dont les marges sont inférieures à $2500 \mathrm{~F} / \mathrm{ha}$.

$\mathrm{Au}$-delà de cette solution, et sur un plan plus général, l'interrogation pour ces grands troupeaux est de savoir si on peut, dans les conditions françaises, miser sur un modèle également peu intensif au niveau de l'animal, dans lequel, en contrepartie d'un travail par brebis réduit mais bien ciblé, on obtiendrait une marge moyenne grâce à des charges très réduites et non par un produit élevé (« à la britannique »). Faut-il rechercher dans cette voie, même si pour l'instant une telle orientation va quelque peu à l'opposé de nos observations qui montrent que, au-delà de 450 à 500 brebis, c'est la marge par brebis qui est le premier facteur du revenu et non les effectifs par travailleur - notamment à cause des charges de structures... Une autre limite va également à l'encontre, celle du plafonnement des aides compensatoires (contrairement à la GrandeBretagne), maintenant porté à 50 UGB, seuil encore trop bas. En outre, et c'est le plus important, une telle extensification animale amplifierait la saisonnalité de la production, en la rapprochant de celle des Britanniques et Irlandais, le contraire de ce que veut faire la filière ovine française.

Ce sont toutes ces raisons qui nous incitent à concevoir, pour l'instant, une extensification possible de l'usage des sols sans perdre sur la productivité du troupeau, qu'il soit ovin ou bovin; idée qui sous-tend le protocole de " l'expérimentation système » mis en place au domaine INRA de Redon (de Montard et al 1990). Les Herbagers Autonomes avec qui nous dialoguons le pensent aussi.

\section{Remerciements}

Nous remercions les agriculteurs qui ont accepté de travailler avec nous ainsi que nos collègues de l'INRA et les ingénieurs professionnels régionaux qui nous ont aidés à mettre en place cet observatoire, ceux de la Chambre d'Agriculture, S. Lafarge alors animatrice du RNED ovin et actuellement du GEHP, le CGER (L. Rétif) avec qui nous avons une collaboration particulière, l'ATPIR Poitou Charente pour son appui.

\section{Références bibliographiques}

BENOT M., LAIGNEL, G., LIENARD G., 1989. Economie des Elevages ovins de montagne. Analyse d'un échantillon d'exploitations du Massif Central Nord (Année 1987). Publ. Economie Elevage, INRA-Theix, 38 p. + Annexe.

BENOIT M., LAIGNEL G., LIENARD G., 1990. Résultats des élevages ovins du Montmorillonnais. Campagne 1990 et évolutions. Non publié.

BLANCHEMAIN A., 1988 . Intensification, extensification, quel avenir pour la production ovine française. Economie Rurale, n" 183 , pp. 26-34.

BOUTONNET J.P., MARTINAND P., 1979. Intensification de la production et marché mondial de la viande ovine, contradictions propres à la France. INRA - ESR Montpellier, Série Etude n" 47

BOUTONNET J.P., TCHAMITCHIAN L., 1990. Haute productivité chez les ovins: avantages et inconvénients. Communication à la 41e Réunion de la FEZ. Economie et Sociologie Rurale, IRA - Montpellier, $16 \mathrm{p}$.

CEMAGREF, Division «Techniques et économies des exploitations d'élevages bovins et ovins ", 1989. Note sur la démarche d'analyse des exploitations agricoles pratiquée par la Division TEEBO du CEMAGREF. CEMAGREF Riom. Doc de travail, 52 p. + annexe.

CHAALALI A., 1990. La production de viande ovine en France. Contribution à l'étude des structures: typologie et analyses comparatives. Publ. Labo Economie Rurale de Grignon, INRA., Etudes et Recherches n" 8, 395 p. + annexe.

CGER de la Vienne, 1989. Résultats Economiques 1988. Poitiers, $230 \mathrm{p}$.
DE MONTARD F.X., LOUAULT F., THERIEZ M., BRELURUT A PAILLEUX Y, BENOIT M, LIENARD G, 1990. Comment concilier une utilisation plus extensive du territoire et un élevage ovin performant ? Premiers résultats d'une expérience en Massif Central Nord (In « L'extensification, une forme moderne de modernisation - Séminaire Dijon - Octobre 1990 " - Edition CIFAR - Paris, pp 93-108.).

DURAZO F., LOSSOUARN J., 1990. L'extensif : quelles perspectives pour les bovins et les ovins en Bretagne Centrale. CERFOPA - INA - Paris.

FENELON J.P., 1989. Qu'est ce que l'analyse des données ? Edit. LEFONEN, 311 p.

HUBERT B., 1990. Réaménagement du périmètre incendié de pins maritimes Cévenol : un exemple de gestion et de protection de l'espace en relation avec les systèmes extensifs. INRA - SAD (In " l'extensification, une forme moderne de modernisation " - Séminaire Dijon. Octobre 1990, Edition CIFAR Paris, pp. 75-92).

ITOVIC, ITEB, ENITA, 1990. Des exemples d'exploitations bovines et ovines valorisatrices d'espaces en zones défavorisées (In "l'extensification, une forme moderne de modernisation »-Séminaire Dijon. Octobre 1990, Edition CIFAR Paris, pp. 75-92).

JEAN Y., 1985. Intensification-extensification ou diversité des systèmes de production ovine dans le Montmorillonnais. Economie Rurale n"175, p. 36-43.

RNED Ovin. Fiches systèmes pour le Poitou-Charente. 


\section{Summary}

Should sheep farmers in the Montmorillonnais area (Vienne, France), choose intensification or should they opt for more extensive methods? Observations to fuel a debate.

Due to fierce competition from Great Britain and Ireland, French sheep farming has been going through a difficult period market by a continual increase in imports and a decrease in prices paid to farmers $(-\mathbf{4 0} \%$ between 1980 and 1990). Intensive high input sheep farming has come under fire but it is possible to replace it, and if so, under what conditions, by less intensive systems?

This study deals with sheep farms in the Montmorillonnais are (Vienne, France) which have relatively large flocks for French conditions $(300$ ewes and 430 lambs per farm worker). Seven different production systems were identified. Two reasonably successful approaches differing in their emphasis on intensification were compared. The « successful intensives » use a system which enables them to produce both cash crops and sheep, thanks to a high density on grazing areas $\mathbf{( 1 . 5}$ livestock units/hectare) and the fattening of $2 / 3$ of the lambs in the sheep sheds, the whole system requiring high input of fertilizers (110 units of $\mathbf{N}+\mathbf{P}+\mathrm{K} /$ hectare) and consumption of concentrate $(190 \mathrm{~kg} / \mathrm{ewe})$. « The self sufficiency graziers » opted for a system relying more heavily on grass grazing: $3 / 4$ of the lambs are put out to graze and there is a lower density of sheep in grazing areas (1 livestock unit/ha). There is also a low input of fertilizers (58 NPK/ha) and consumption of concentrate (107 kg of concentrates/ewe). However almost the whole area farmed is given over to sheep and slightly more lambs are produced (430 as compared with 410 per worker). Also less charges and capital are involved.

The economic results obtained in 1989-1990 are equivalent. But it seems that specialised less intensive farms, are better placed to react to difficulties providing that they are well managed as intensive farms. Specially, it is important to maintain the number of lambs produced by each ewe in order to obtain greater profits stemming from reduced expenses.

BENOIT M., LAIGNEL G., LIENARD G., 1991. L'élevage ovin Montmorillonnais partagé entre l'intensification et une voie plus extensive. Observations pour un débat. INRA Prod. Anim., 4 (5), 343-359. 


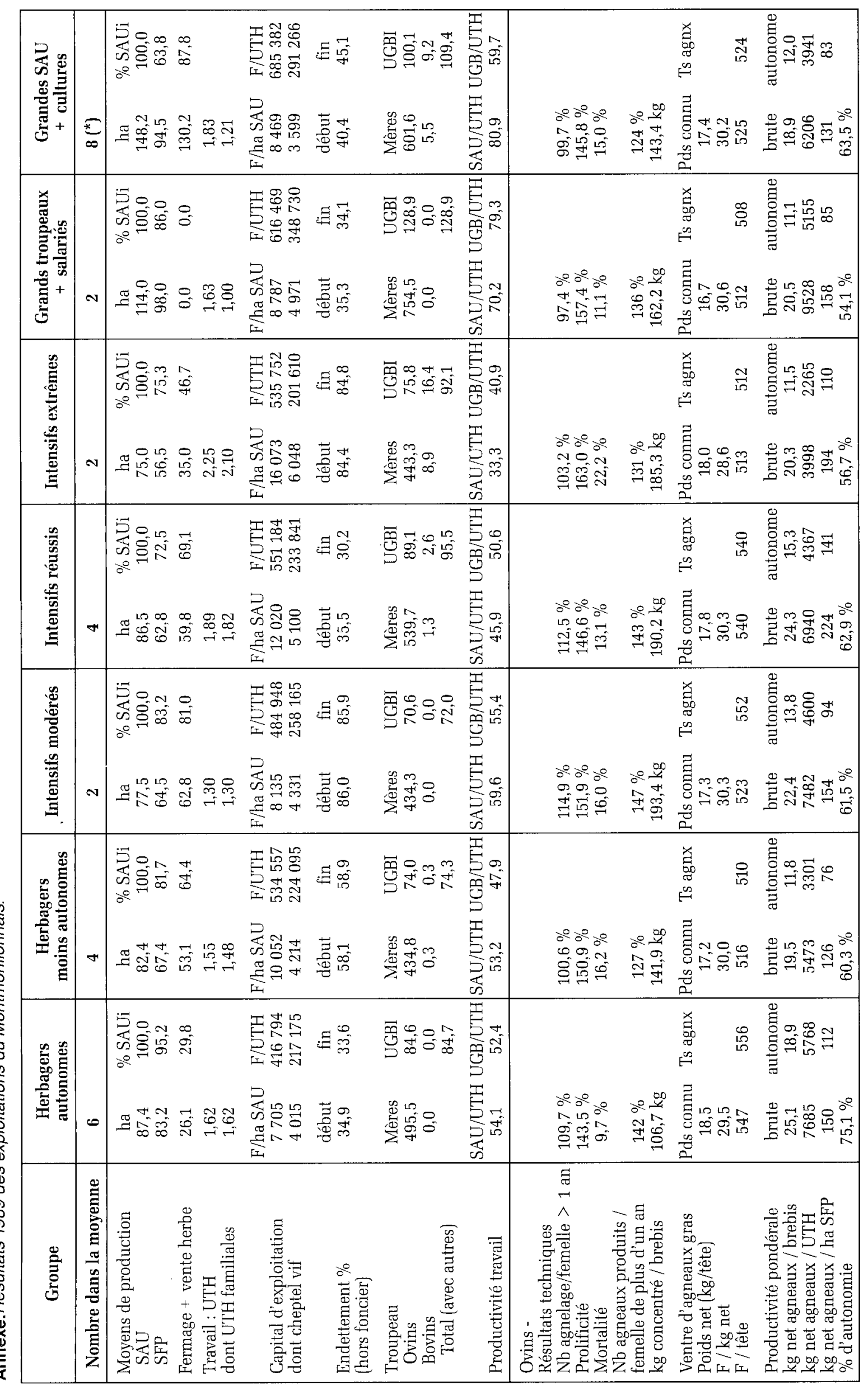




\begin{tabular}{|c|c|c|c|c|c|c|}
\hline 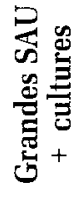 & $\underset{\infty}{\infty}$ & 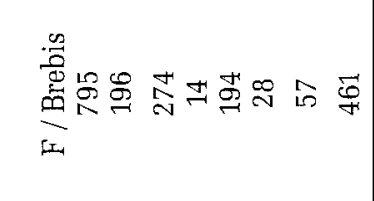 & m & $\begin{array}{ll}\stackrel{g}{N} & \stackrel{N}{N} \\
= & 0 \\
m\end{array}$ & 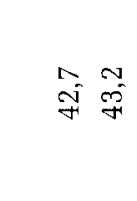 & $\begin{array}{ll}1 & 0 \\
\infty & 0 \\
\infty & \infty \\
N & +\end{array}$ \\
\hline 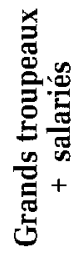 & $\mathbf{N}$ & 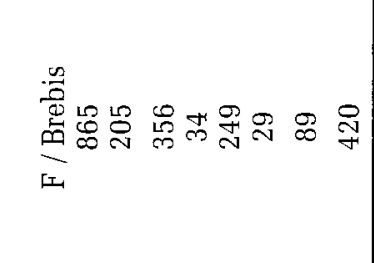 & ○ N & 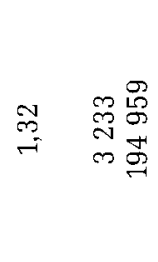 & $\begin{array}{l}\infty \\
\text { si } \\
m^{-}\end{array}$ & 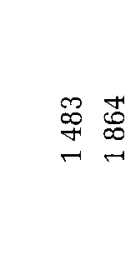 \\
\hline 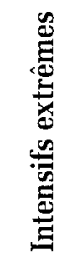 & $\sim$ & 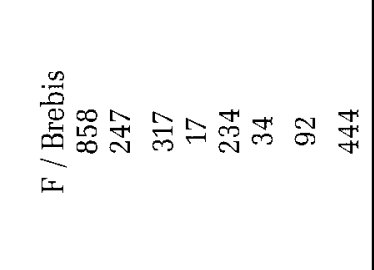 & 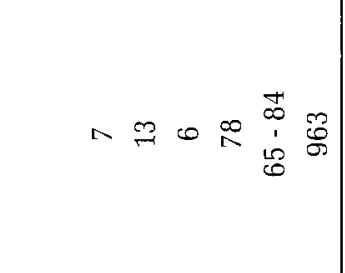 & 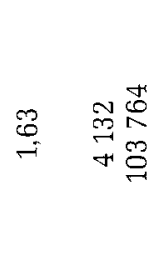 & $\begin{array}{ll}0 & 0 \\
f^{+} & 00^{\circ}\end{array}$ & 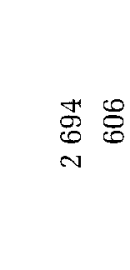 \\
\hline 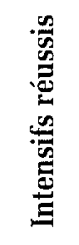 & + & 营 & 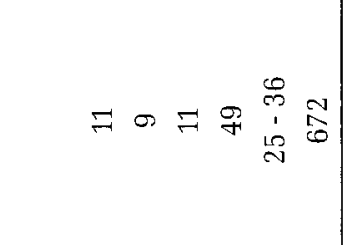 & 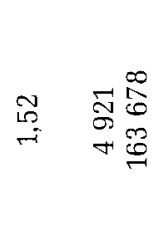 & $\vec{r}$ if & 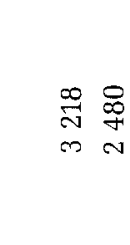 \\
\hline 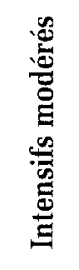 & $\mathrm{N}$ & 乐 & ○ & 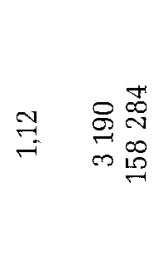 & $\begin{array}{ll}2 & 0 \\
\sigma^{-} & 18 \\
8\end{array}$ & 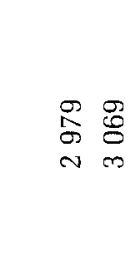 \\
\hline 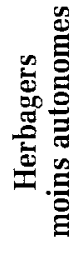 & $r$ & 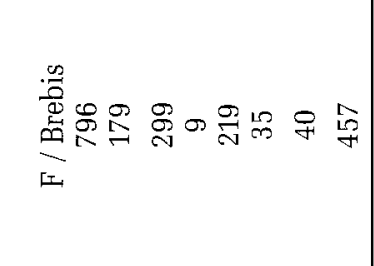 & $N+\vec{m}=\begin{array}{cc}\hat{N} & 0 \\
\frac{d}{d} & 0\end{array}$ & 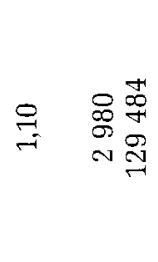 & $\begin{array}{l}\text { or } \\
\dot{f} \\
\vec{f}\end{array}$ & 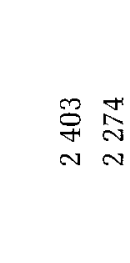 \\
\hline 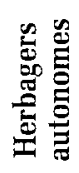 & 0 & 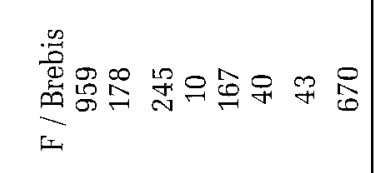 & 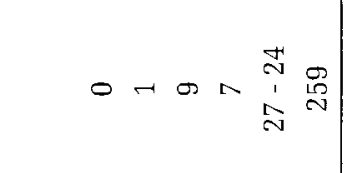 & 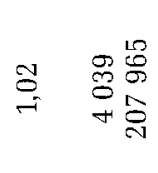 & 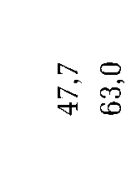 & 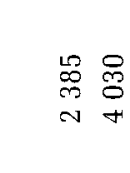 \\
\hline 菷 & 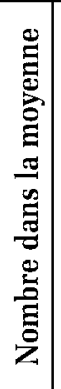 & 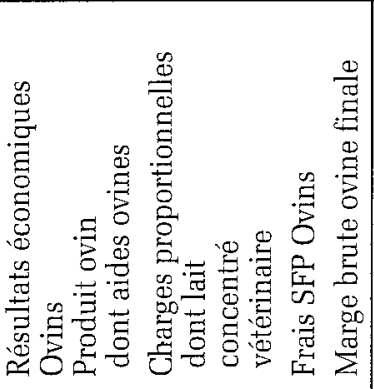 & 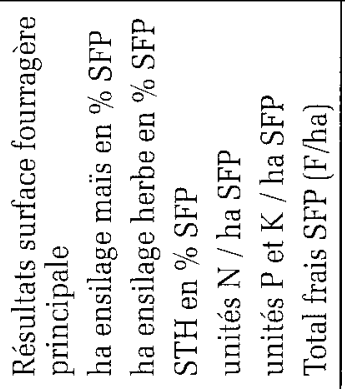 & 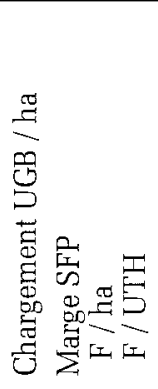 & \multicolumn{2}{|c|}{ 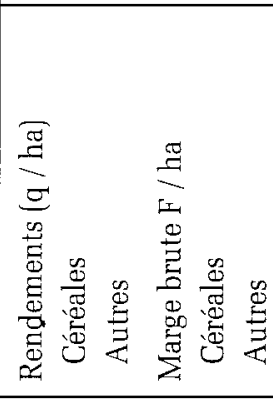 } \\
\hline
\end{tabular}




\begin{tabular}{|c|c|c|c|c|c|c|c|c|c|}
\hline 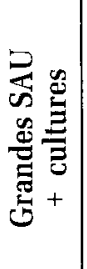 & $\underbrace{\pi}_{\infty}$ & 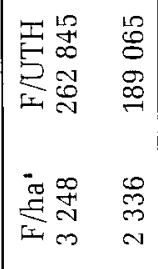 & 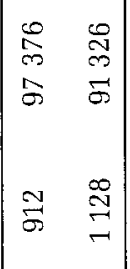 & 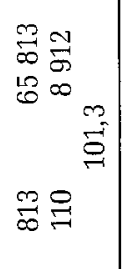 & 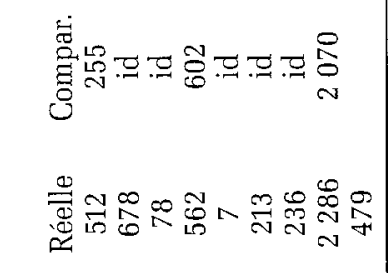 & 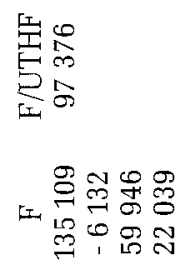 & 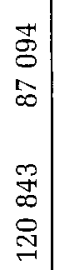 & 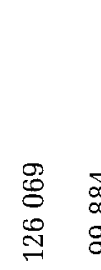 & 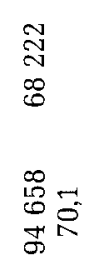 \\
\hline 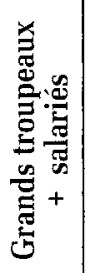 & N & 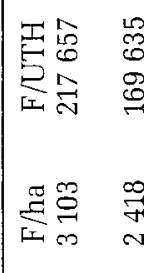 & 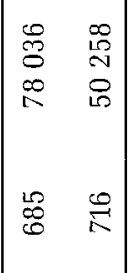 & 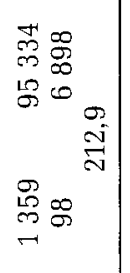 & 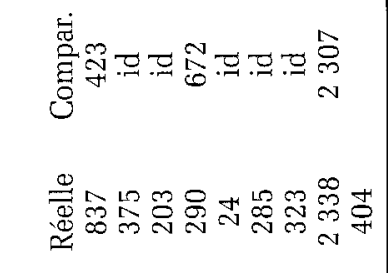 & 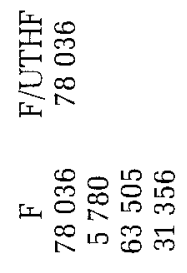 & 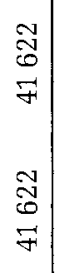 & $\begin{array}{l}\text { P } \\
\text { m } \\
0 \\
0 \\
10\end{array}$ & 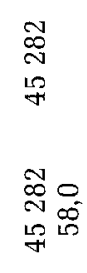 \\
\hline 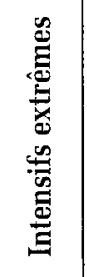 & $\boldsymbol{N}$ & 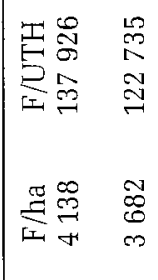 & 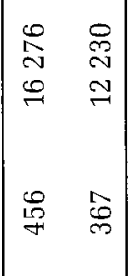 & 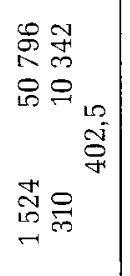 & 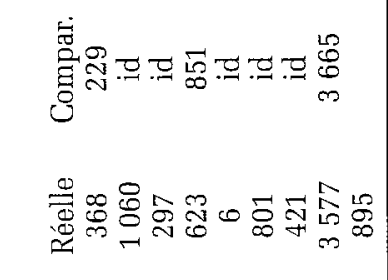 & 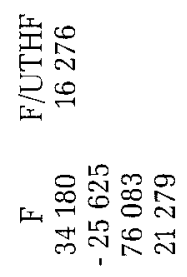 & $\begin{array}{l}8 \\
8 \\
0 \\
20 \\
10\end{array}$ & $\begin{array}{l}\vec{F} \\
\mathscr{8}\end{array}$ & 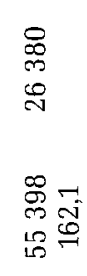 \\
\hline 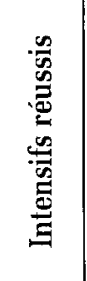 & + & 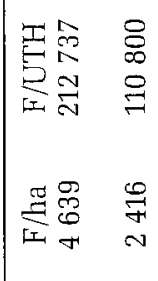 & 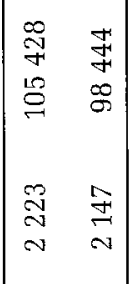 & 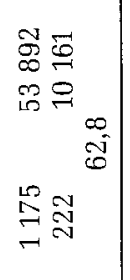 & 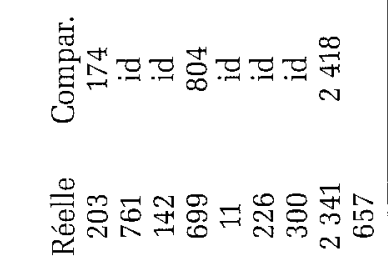 & 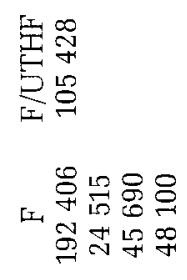 & 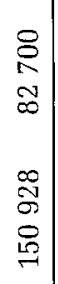 & $\begin{array}{l}\mathscr{D} \\
\infty \\
\stackrel{0}{0} \\
\stackrel{0}{\varrho}\end{array}$ & 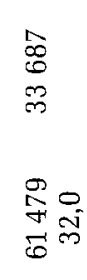 \\
\hline 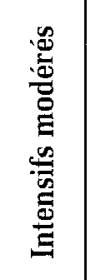 & $N$ & 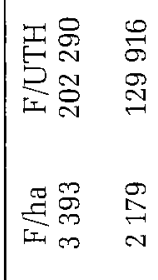 & 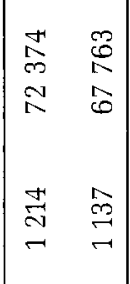 & 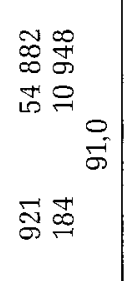 & 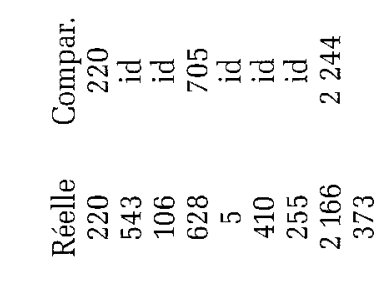 & 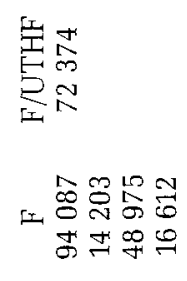 & $\begin{array}{l}\mathbb{N} \\
\stackrel{8}{S} \\
\stackrel{8}{N}\end{array}$ & $\begin{array}{ll}0 & 0 \\
0 & \\
10 & \\
0 & 7\end{array}$ & 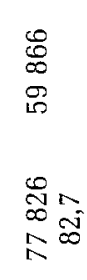 \\
\hline 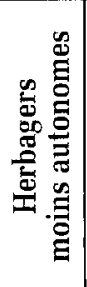 & 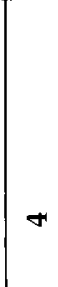 & 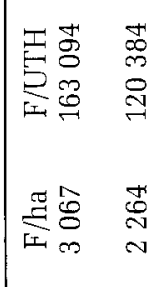 & 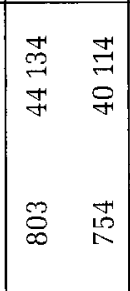 & 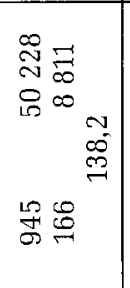 & 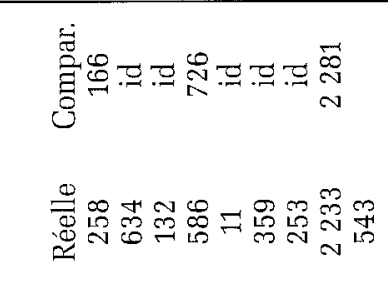 & 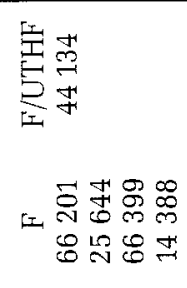 & 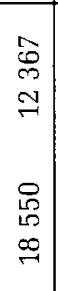 & $\begin{array}{ll}\infty & 0 \\
1 & 0 \\
1 & 0 \\
0 & 0\end{array}$ & 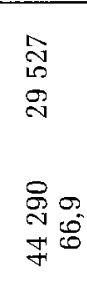 \\
\hline 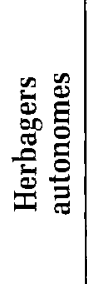 & 0 & 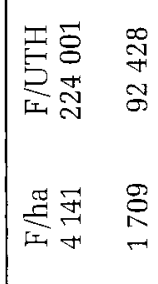 & 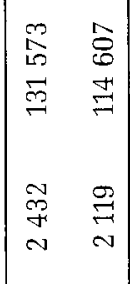 & 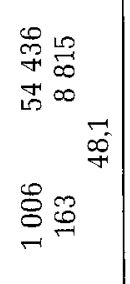 & 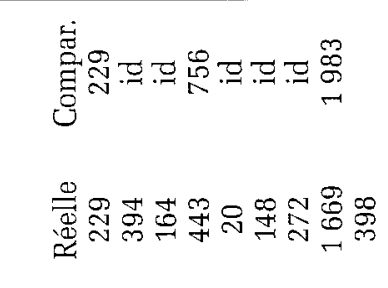 & 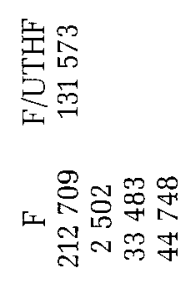 & 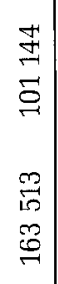 & $\begin{array}{ll}\infty & 0 \\
\infty & 0 \\
\infty & 0 \\
\text { के }\end{array}$ & 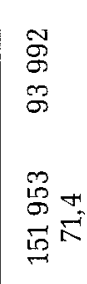 \\
\hline 节 & 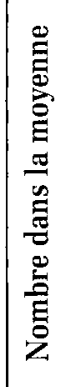 & 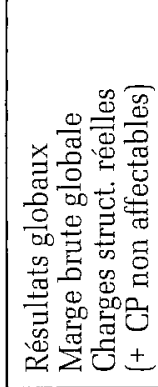 & 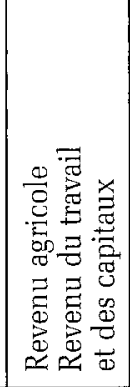 & 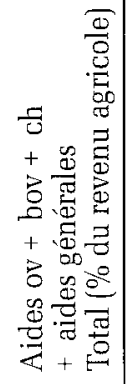 & 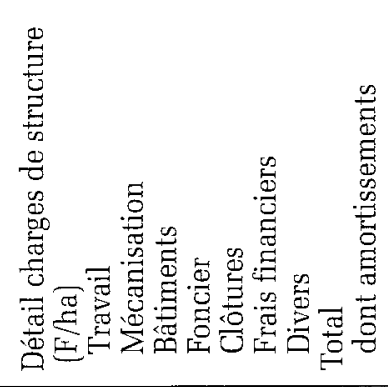 & 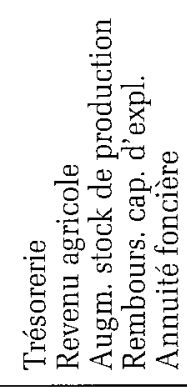 & \begin{tabular}{c|} 
\\
0 \\
0 \\
0 \\
0 \\
0 \\
0 \\
0 \\
0
\end{tabular} & 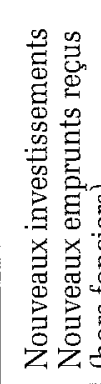 & 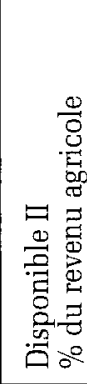 \\
\hline
\end{tabular}

